\title{
Independent and Combined Effects of Sex and Biological Maturation on Motor Coordination and Performance in Prepubertal Children
}

Leonardo G. O. Luz

LACAPS, Federal University of Alagoas, Brazil

Sean P. Cumming

School for Health, University of Bath, UK

João P. Duarte, João Valente-dos-Santos,

Maria J. Almeida, Aristides Machado-Rodrigues, and

Cristina Padez

University of Coimbra, Portugal

Bruno Cleiton M. Carmo

LACAPS, Federal University of Alagoas, Brazil

Rute Santos

Early Start Research Institute, Faculty of Social Sciences, University of Wollongong, Australia; CIAFEL, University of Porto, Portugal

André Seabra

CIAFEL, University of Porto, Portugal

Manuel J. Coelho-E-Silva

University of Coimbra, Portugal

Corresponding Author:

Manuel J. Coelho-E-Silva, Estádio Universitário de Coimbra, Pavilhão III, Coimbra 3040-156, Portugal. Email: mjcesilva@ hotmail.com

\begin{abstract}
Sex differences and maturation-associated variation in fitness and motor coordination were examined in children aged 8-9 years (n 1/4 128, 67 girls). Assessments included stature and body mass, two-component body composition, percentage of predicted adult stature (as an index of biological maturation), and motor performance and coordination (Körperkoordinationstest für Kinder). Compared to girls, boys were less advanced in maturation status, possessed larger fat mass, demonstrated superior performances in six tests of fitness, and obtained one superior score on the Körperkoordinationstest für Kinder. After controlling for somatic maturation, sex differences persisted in the two multivariate domains: motor performance and motor coordination.
\end{abstract}

Keywords

childhood, motor development, KTK battery, coordination 


\section{Introduction}

The health-related benefits of regular physical activity and physical fitness in children and adolescents have been widely documented (Strong et al., 2005; see Physical Activity Guidelines Advisory Committee, 2008). More specifically, regular participation in physical activity has been consistently associated with enhanced cardiovascular, metabolic, skeletal, and mental health, and a healthy weight status (Janssen \& Leblanc, 2010). Motor competence and the processes of growth and maturation have been shown to contribute to engagement in physical activities during childhood and adolescents (Robinson et al., 2015; Smart et al., 2012). Accordingly, relations among these constructs are of particu- lar interest to those involved in the study and promotion of physical activity and health in youth (Cumming, Sherar, Esliger, Riddoch, \& Malina, 2014; Lopes, Stodden, Bianchi, Maia, \& Rodrigues, 2012).

Motor competence is a global term used to reflect various terminologies that have been used in previous literature (i.e., motor proficiency, motor performance, funda- mental movement/motor skill, motor ability, and motor coordination) to describe goal-directed human movement (Robinson et al., 2015). While motor competence typically improves with age, maturity, and experience, children of the same chrono- logical age and/or maturation status can demonstrate significant variance in motor competence. Several studies in young people have suggested a positive association between motor competence and several health and behavioral outcomes (Burns et al., 2009; Cairney, Hay, Veldhuizen, \& Faught, 2010; Krombholz, 2013; Luz et al., 2015; Martins et al., 2010; Robinson et al., 2015). Physical activity and sports participation have a reciprocal relationship with motor competence through- out childhood and adolescence (Graham, Sirard, \& Neumark-Sztainer, 2011; Okely, Booth, \& Patterson, 2001; Rivilis et al., 2011; Robinson et al., 2015). Accordingly, the development and refinement of motor skills in young people should be con- sidered a key objective in educational curricula and public health policies.

Between the ages of 3 and 6 years, sex differences in motor competence are minimal, as both boys and girls demonstrate marked improvements across a range of motor skills (Kakebeeke, Locatelli, Rousson, Caflisch, \& Jenni, 2012). During this time period, girls often outperform boys on balance and coordination tasks, such as hopping and skipping. From mid-to-late childhood and throughout adolescence, however, boys, compared to girls, demonstrate greater improvements in skills requiring speed, strength, and power, 
resulting in notable sex differences in motor competence. While much of these differences can be attributed to sex-associated differences in pubertal development (i.e., greater gains in absolute, and relative lean mass in boys), the differences that emerge during late childhood and continue through adolescence are more likely to reflect gender differences in socialization and/or the provision of opportunity (i.e., greater encouragement and opportunity for boys).

It is important to consider inter-individual variability in biological maturation and control for this variable in pediatric exercise research, especially in studies involving the functional and performance characteristics of young athletes (Baxter-Jones, Eisenmann, \& Sherar, 2005). Biological maturation can be viewed from two perspectives, status and timing. The former refers to the state of maturation at the time (chronological age) of observation, while the latter refers to the chronological age at which specific maturational landmarks (e.g., menarche, puber- tal onset) are attained. The most common indicators of maturity status are skeletal age and development of secondary sex characteristics.

One commonly adopted indicator of maturity timing is age at peak height velocity (PHV). This method requires longitudinal data to obtain accurate esti- mates of age at PHV for individuals. Secondary sex characteristics - breasts and pubic hair in girls or genital and pubic hair in boys - are also widely used in the literature (Baxter-Jones et al., 2005), and at times incorrectly. Given the sensitivity of evaluating pubertal status, especially when using self-report, the issue of the reproducibility of assessments and the potential estimation errors needs to be considered when using sex secondary characteristics to assess mat- uration (Rasmussen et al., 2015). Allowing for potential error in assessment, stages of puberty have several limitations. First, stages are discrete categories imposed on a continuous process of maturation, and, consequently, a youngster is either in a stage or not in a stage; there are no intermediate stages. Second, duration of a stage and age at transition from one to another are difficult to estimate; to do so, longitudinal observations at relatively short intervals are required. Finally, the rate of transition from pubertal onset to maturity is highly variable among individuals and is not extensively documented (Malina, Bouchard, \& Bar-Or, 2004) as compared to secondary sex characteristics.

Somatic maturation can be estimated as a percentage of predicted adult stature attained at measurement, if data pertaining to the height of the individual's biological parents is available (Baxter-Jones et al., 2005). Percentage of predicted mature stature (PMS) at a given age had moderate concordance with skeletal age in male youth participants in 
American football (Malina, Dompier, Powell, Barron, \& Moore, 2007) and soccer (Malina, Coelho e Silva, Figueiredo, Carling, \& Beunen, 2012), and it provides a reasonably equivalent range of distribution when classifying individuals as early-maturing, on time, or latematuring.

The degree to which biological maturation contributes to sex differences in motor competence is unclear as yet, and may vary relative to developmental stage (i.e., early or late childhood, adolescence). Whereas advanced maturation is generally associated with improved motor competence in early childhood, it cannot explain why boys, who typically mature later than girls, demonstrate superior motor performance in late childhood. Furthermore, advanced maturity may differentially affect motor competence in boys and girls during adolescence due to sex-related differences in pubertal development. While advanced matur- ation in boys is generally associated with greater functional and motor compe-tence, advanced maturity in girls is associated with poorer performance, particularly in skills that require elements of gross motor control and movement and/or endurance (Malina et al., 2004). Biological maturation, rather than chronological age, seems a major correlate of inter-individual variability in physical activity (Cumming, Standage, Gillison, \& Malina, 2008; Sherar, Esliger, Baxter-Jones, \& Tremblay, 2007). Studies reporting the effect of biological mat- uration on motor behavior are still limited (Sherar, Cumming, Eisenmann, Baxter-Jones, \& Malina, 2010) and are predominantly focused in adolescent years (Malina et al., 2004). This study was aimed to investigate the independent and combined effects of sex and biological maturation on motor competences assessed using a physical fitness battery in addition to four motor coordination tests among pre-pubertal children within a single age group.

\section{Method}

\section{Sample}

This sample did not pretend to represent the population of Arapiraca and was recruited to examine the hypothesis of the current study. Participants aged 8.0-8.9 years. This narrow age range was chosen to limit the effect of chrono- logical age as a source of inter-individual variability, and the band of eight years corresponds to pre-menarche status (Malina, 2014). The final sample included 128 children (67 girls, 61 boys). 


\section{Procedure}

This was a school-based cross-sectional study carried out in four primary schools (two public schools and two private schools) from the area of Arapiraca, a city in the Northeast state of Brazil. The study was conducted in accordance with the Declaration of Helsinki for human studies of the World Medical Association. The ethics committee of the Federal University of Alagoas (Brazil) approved the study (CAAE 09200413.5.0000.5013). Prior to data col- lection, parents or legal guardians signed informed consent (response rate 90\%). In addition, verbal consent was obtained from participants after the presenta- tion of the aim and procedures. Participants were informed that their inclusion was voluntary and that they could withdraw from the study at any time. Data were collected in the schools during weekdays corresponding to visits of the research team. A total of three sessions were needed for each class. All visits were performed within a single month. The first visit was used for collection of personal data and anthropometry. A second one was used to assess physical fitness items, and the final session was used to evaluate motor coordination (Korperkoordinationstest fur Kinder (KTK) battery), obtaining missing data. All groups followed the same order of tests except for KTK that was organized based on a circuit rotation without visual contact between participants.

\section{Anthropometry}

An experienced observer measured all participants, adopting a standardized protocol (Lohman, Roche, \& Martorell, 1988). Stature was measured to the nearest $0.1 \mathrm{~cm}$ using portable stadiometer (Sanny Caprice, Sao Paulo, Brazil). Body mass was measured to the nearest $0.1 \mathrm{~kg}$ with a digital scale (Techline, Sao Paulo, Brazil). Skinfold thickness was assessed to the nearest $\mathrm{mm}$ using Lange skinfold callipers (Beta Technology, Santa Cruz, California, USA) in the fol- lowing references: triceps, biceps, subscapular, suprailiac, abdominal, and calf. Estimates of fat mass percentage were obtained from sexspecific equation derived from the sum of the triceps and subscapular skinfolds (Slaughter et al., 1988). Afterward, estimated fat and fat-free masses were calculated to the nearest $0.1 \mathrm{~kg}$. Repeated measurements were obtained on 19 students within one week, and technical errors of measurements were determined: stature, $0.6 \mathrm{~cm}$; body mass, $0.6 \mathrm{~kg}$; skinfolds, $1.0-1.4 \mathrm{~mm}$.

\section{Biological maturation}

The Khamis-Roche method (Khamis \& Roche, 1994) was used to PMS. The protocol requires decimal age, stature, and body mass of the participant and average parental stature. The stature of the parents was collected through the identity card. This protocol was developed on children from the Fels Longitudinal Study conducted in South Central Ohio in the United States. Current stature was expressed as a percentage of PMS (\%PMS). It is assumed that among children of 
the same chronological age, individuals closer to the estimated mature stature are more advanced in biological maturation compared

with individuals who are further from estimated mature stature (Malina et al., 2004). The Khamis-Roche method has been employed as an estimate of biological maturity status in several studies (Cumming, Standage, Gillison, Dompier, \& Malina, 2009; Malina, Cumming, Morano, Barron, \& Miller, 2005) and has been validated against an established indicator of maturity (skeletal age) in youth American football players (Malina et al., 2007). The mean error bound (median absolute deviation) between actual and PMS at 18 years of age was $2.2 \mathrm{~cm}$ in boys and $1.7 \mathrm{~cm}$ in girls (Khamis \& Roche, 1994). To evaluate the maturity status, the \%PMS was expressed as z score on the mean and standard deviation by sex and age of the Berkeley Guidance Study sample, University of California (Bayer \& Bayley, 1959). The $\mathrm{z}$ scores of the \%PMS are often used to estimate the maturity status. Separately for males and females, two groups contrasting in somatic maturation were derived from $\mathrm{z}$ scores of attained \%PMS: earliest maturing $(\mathrm{P}<50 \%)$ and latest maturing $(\mathrm{P}>50 \%)$. This approach was previously operated for Flemish soccer players aged 11-14 years (Deprez et al., 2014).

\section{Physical fitness}

Fitness was assessed according to standard protocols using the following items of the EUROFIT test battery (Committee for the Development of Sports, 1988): standing long jump (SLJ; lower body functional strength), sit-and-reach (SAR; flexibility), handgrip strength (HGP; static strength), 105-m shuttle run (SHR; agility), and 20-m endurance shuttle run (ESR; cardiorespiratory endurance). In addition, 60-s sit-ups (SUP; trunk strength), 2-kg medicine ball throw (2-kg BT; upper body functional strength), and 25-m dash (SPR; velocity) were also mea- sured. The detailed description of the tests protocols and procedures can be seen elsewhere (Committee for the Development of Sports, 1988). The selection of the tests was aligned with available literature on school children combining fitness and motor coordination (Vandendriessche et al., 2011).

\section{Motor coordination}

Motor coordination was evaluated using a 4-item battery, the KTK (Kiphard \& Schilling, 1974). The battery is often used in primary school children in Flanders (D'Hondt et al., 2011; Vandendriessche et al., 2012), Germany (Krombholz, 2006), and Portugal (Lopes, Santos, Pereira, \& Lopes, 2013; Lopes et al., 2012). It includes four items that were conducted in the following order: walking backward on balance beams (WB), moving sideways on boxes (MS), jumping sideways across a wooden slat (JS), and hopping for height on one leg (HH) (Camacho-Araya, Woodburn, \& Boschini, 1990). Metric 
properties of KTK (Kiphard \& Schilling, 1974) showed reasonable test-retest reliability (coefficients ranged between 0.80 and 0.96 ).

\section{Statistical analysis}

Descriptive statistics (range, means, standard errors, standard deviations, and $95 \%$ confidence intervals) were calculated for total sample. Kolmogorov-Smirnov was used to test normality, and appropriate $\log$ transformations $(\log 10)$ were adopted to normalize distributions on three physical fitness tests (HGP, SHR, and ESR) and two KTK items (JS and MS). Sex differences were examined based on the determination of mean differences and respective magnitude effect in parallel to $t$ tests. Subsequently, Pearson correlations examined bivarivate associations between biological maturation assessments (percentage of mature stature attained and $\mathrm{z}$ score for percentage of mature stature attained) and motor scores (physical fitness tests and KTK items) for the total sample and also separately for boys and girls. The correlation coefficients were interpreted as follows (Hopkins, Marshall, Batterham, \& Hanin, 2009): trivial $(\mathrm{r}<0.1)$, small $(0.1<\mathrm{r}<$ $0.3)$, moderate $(0.3<\mathrm{r}<0.5)$, large $(0.5<\mathrm{r}<0.7)$, very large $(0.7<r<0.9)$, and nearly perfect $(r>0.9)$.

Descriptive statistics (means, standard deviations, mean differences) were calculated within each sex for groups contrasting in somatic maturation given by $\mathrm{z}$ score of attained PMS for anthropometric measures, physical fitness tests, and KTK items. Then, groups were compared using the two-sided t test, and standardized differences between means were reported using Cohen's $d$ values interpreted as follows: $<0.20$ (trivial), 0.20 to 0.59 (small), 0.60 to 1.19 (moder- ate), 1.20 to 1.99 (large), 2.0 to 3.9 (very large), and >4.0 (extremely large) (Hopkins et al., 2009).

Further, the independent and combined effects of sex and maturity status on motor scores were examined using multivariate analysis of variance (MANOVA), separately for physical fitness tests and KTK items. Finally, the effect of sex (boys and girls) on physical fitness tests and KTK items scores was examined using a multivariate analysis of covariance (MANCOVA) ( $\mathrm{z}$ score of attained PMS as covariate). When MANOVA or MANCOVA detected a statistically significant effect, subsequent analysis of variance (ANOVA) and ana- lysis of covariance were used to detect the contribution of the single dependent variables to the multivariate solution. Data were analyzed using IBM SPSS 22.0 (SPSS, Inc., Chicago, IL). Significance level was set at $5 \%$ for all inferential statistics.

\section{Results}

Descriptive characteristics of the sample are presented in Tables 1 and 2. Girls showed higher values for attained PMS than boys. Boys presented higher mean values for estimated fat-free mass and attained better performances on 2-kg medicine BT, HGP, 60-s SUP, SLJ, 25-m dash, 10 5-m agility, and $\mathrm{HH}$.

As presented in Table 3, correlations between somatic maturation given by attained percentage mature stature and motor performance items were moderate 
Table 1. Descriptive statistics (range, mean value including standard error of the mean and 95\% confidence limits of the mean, and standard deviation) and test for normality for the total sample of school children aged 8.0-8.9 years ( $\mathrm{N} \mathrm{1/4} 128 ; 61$ boys, 67 girls).

\begin{tabular}{|c|c|c|c|c|c|c|c|c|}
\hline \multirow[b]{2}{*}{$\mathrm{X}_{\mathrm{i}}$ : Variables } & \multicolumn{2}{|c|}{ Range } & \multirow[b]{2}{*}{ Mean } & \multirow[b]{2}{*}{ SEM } & \multicolumn{2}{|c|}{$95 \% \mathrm{CL}$ of the mean } & \multirow[b]{2}{*}{ SD } & \multirow[b]{2}{*}{ Norm } \\
\hline & Min & Max & & & Lower & Upper & & \\
\hline $\mathrm{X}_{1}$ : Chronological age (year) & 8.0 & 8.9 & 8.5 & 0.0 & 8.4 & 8.5 & 0.3 & $0.09 *$ \\
\hline $\begin{array}{l}\mathrm{X}_{2}: \text { Predicted mature } \\
\text { stature }(\mathrm{cm})\end{array}$ & 147.7 & 189.1 & 169.6 & 0.8 & 168.0 & 171.2 & 9.1 & 0.07 \\
\hline $\mathrm{X}_{3}$ : Attained PMS (\%) & 72.1 & 87.5 & 77.5 & 0.3 & 76.9 & 78.2 & 3.5 & $0.08^{*}$ \\
\hline $\mathrm{X}_{4}: \mathrm{z}$ score of APMS & 3.2 & 5.5 & 0.3 & 0.1 & 0.0 & 0.5 & 1.5 & 0.05 \\
\hline $\mathrm{X}_{5}:$ Stature of the father $(\mathrm{cm})$ & 152.5 & 189.0 & 171.3 & 0.7 & 169.9 & 172.6 & 7.9 & $0.18 \mathrm{z}$ \\
\hline $\begin{array}{l}\mathrm{X}_{6}: \text { Stature of the } \\
\text { mother }(\mathrm{cm})\end{array}$ & 149.5 & 179.3 & 165.1 & 0.5 & 164.2 & 166.0 & 5.4 & 0.05 \\
\hline $\begin{array}{l}\mathrm{X}_{7} \text { : Midparental } \\
\text { stature }(\mathrm{cm})\end{array}$ & 142.5 & 176.0 & 158.9 & 0.6 & 157.8 & 160.1 & 6.7 & $0.09 *$ \\
\hline $\mathrm{X}_{8}:$ Stature $(\mathrm{cm})$ & 119.0 & 146.2 & 131.3 & 0.5 & 130.3 & 132.4 & 5.8 & 0.04 \\
\hline $\mathrm{X}_{9}$ : Body mass (kg) & 18.1 & 61.7 & 31.5 & 0.7 & 30.2 & 32.8 & 7.4 & $0.08 *$ \\
\hline $\mathrm{X}_{10}$ : Fat mass $(\%)$ & 8.5 & 65.0 & 27.1 & 0.8 & 25.6 & 28.6 & 8.6 & $0.11 *$ \\
\hline $\mathrm{X}_{11}$ : Fat mass $(\mathrm{kg})$ & 1.5 & 40.1 & 9.1 & 0.5 & 8.1 & 10.0 & 5.3 & $0.13 z$ \\
\hline $\mathrm{X}_{12}:$ Fat-free mass $(\mathrm{kg})$ & 15.7 & 30.3 & 22.5 & 0.3 & 21.9 & 23.0 & 3.2 & 0.05 \\
\hline $\mathrm{X}_{13}: 2$ - $\mathrm{kg}$ ball throw $(\mathrm{cm})$ & 113 & 327 & 190 & 3 & 184 & 196 & 35 & 0.07 \\
\hline $\begin{array}{l}\mathrm{X}_{14} \text { : Handgrip } \\
\text { strength }(\mathrm{kg} \mathrm{f})\end{array}$ & 5.5 & 25.0 & 13.5 & 0.3 & 12.8 & 14.1 & 3.8 & $0.12 \mathrm{z}$ \\
\hline $\mathrm{X}_{15}: 60$-s sit-ups & 6 & 36 & 16.6 & 0.8 & 15.0 & 18.3 & 9.4 & 0.08 \\
\hline $\mathrm{X}_{16}$ : standing long jump $(\mathrm{cm})$ & 32 & 198 & 92 & 2 & 88 & 97 & 25 & 0.07 \\
\hline $\mathrm{X}_{17}: 25-\mathrm{m}$ dash $(\mathrm{s})$ & 4.60 & 8.31 & 6.11 & 0.07 & 5.97 & 6.24 & 0.78 & 0.07 \\
\hline $\mathrm{X}_{18}: 10 \quad 5$-m agility (s) & 20.81 & 33.97 & 26.43 & 0.23 & 25.98 & 26.89 & 2.60 & $0.09 *$ \\
\hline$X_{19}: 20-m$ shuttle run $(m)$ & 60 & 880 & 281 & 13 & 256 & 307 & 148 & $0.12 \mathrm{z}$ \\
\hline $\mathrm{X}_{20}:$ Sit-and-reach $(\mathrm{cm})$ & 6.5 & 42.0 & 25.6 & 0.5 & 24.5 & 26.6 & 5.9 & 0.05 \\
\hline $\mathrm{X}_{21}$ : Walking backward & 3 & 68 & 38.2 & 1.2 & 35.9 & 40.6 & 13.5 & 0.07 \\
\hline $\mathrm{X}_{22}$ : Jumping sideways & 6 & 57 & 31.3 & 0.8 & 29.7 & 33.0 & 9.4 & $0.08^{*}$ \\
\hline $\mathrm{X}_{23}$ : Moving sideways & 16 & 80 & 32.0 & 0.7 & 30.7 & 33.3 & 7.6 & $0.14 \mathrm{z}$ \\
\hline $\mathrm{X}_{24}$ : Hopping for height & 0 & 60 & 33.0 & 1.1 & 30.8 & 35.2 & 12.4 & 0.08 \\
\hline
\end{tabular}

PMS: predicted mature stature; APMS: attained predicted mature stature; Norm: normality, KolmogorovSmirnov test with $\mathrm{df}^{1 / 4} 128$.

$* p<0.05 . z p<0.01$.

for girls in 2-kg medicine BT, handgrip, and WB. Among boys, three fitness tests also showed a moderate correlation with somatic maturation: 2-kg medicine BT, HGP, and 20-m SHR. When somatic maturation is given by z score values obtained using age- and sex-specific means and standard deviations extracted from the Berkeley Guidance Study, significant correlations were observed for the 2-kg medicine BT, HGP, 20-m SHR, and WB for girls and for the 20-m SHR, SAR, and WB for boys. 
Table 2. Descriptive statistics (mean standard deviation) by sex and test for equality of means in addition to mean difference (including standard error and 95\% confidence limits) and magnitude effect.

\begin{tabular}{|c|c|c|c|c|c|c|c|c|c|c|c|}
\hline \multirow[b]{2}{*}{ Dependent variables } & \multicolumn{4}{|c|}{ Descriptive statistics } & \multicolumn{3}{|c|}{ Mean difference } & \multicolumn{2}{|c|}{ Magnitude effect } & \multicolumn{2}{|c|}{ Equality of means } \\
\hline & Boys (n & $\left(\mathrm{n}^{1 / 4} 61\right)$ & Girls $\quad(n$ & $(n+1 / 467)$ & Mean & SE & $\begin{array}{c}\text { 95\% CL } \\
\text { (Lower; Upper) }\end{array}$ & $\mathrm{d}$ & (Qualitative) & $\mathrm{t}(\mathrm{df} 1 / 4126)$ & $\mathrm{p}$ \\
\hline Chronological age (years) & 8.5 & 0.3 & 8.4 & 0.3 & 0.1 & 0.1 & $(0.0 ; 0.2)$ & 0.21 & (small) & 1.205 & 0.231 \\
\hline Predicted mature stature $(\mathrm{cm})$ & 176.8 & 5.8 & 163.1 & 6.2 & 13.7 & 1.1 & $(11.7 ; 15.9)$ & 2.31 & (very large) & 13.051 & $<0.01$ \\
\hline Attained PMS (\%) & 74.7 & 1.6 & 80.2 & 2.5 & 5.5 & 0.4 & $(6.3 ; 4.8)$ & 2.62 & (very large) & 14.820 & $<0.01$ \\
\hline Stature $(\mathrm{cm})$ & 132.0 & 5.5 & 130.7 & 6.0 & 1.3 & 1.0 & $(0.7 ; 3.3)$ & 0.23 & (small) & 1.291 & 0.199 \\
\hline Body mass (kg) & 32.6 & 8.0 & 30.5 & 6.8 & 2.1 & 1.3 & $(0.5 ; 4.6)$ & 0.28 & (small) & 1.577 & 0.117 \\
\hline Fat mass $(\%)$ & 25.8 & 10.5 & 28.3 & 6.2 & 2.5 & 1.5 & $(5.5 ; 0.5)$ & 0.29 & (small) & 1.660 & 0.099 \\
\hline Fat mass (kg) & 9.1 & 6.5 & 9.0 & 4.0 & 0.1 & 0.9 & $(1.7 ; 2.0)$ & 0.03 & (trivial) & 0.166 & 0.868 \\
\hline Fat-free mass (kg) & 23.4 & 2.8 & 21.5 & 3.2 & 1.9 & 0.5 & $(0.8 ; 3.0)$ & 0.63 & (moderate) & 3.555 & $<0.05$ \\
\hline 2-kg ball throw $(\mathrm{cm})$ & 204 & $4 \quad 35$ & 177 & $7 \quad 29$ & 27 & 6 & $(16 ; 38)$ & 0.84 & (moderate) & 4.745 & $<0.01$ \\
\hline Hand grip strength (kg.f)* & 14.7 & 4.3 & 12.3 & 2.8 & 2.4 & 0.6 & $(1.1 ; 3.7)$ & 0.67 & (moderate) & 3.758 & $<0.01$ \\
\hline 60-s sit-ups (\#) & 18.5 & 9.5 & 14.9 & 8.9 & 3.6 & 1.6 & $(0.3 ; 6.8)$ & 0.39 & (small) & 2.187 & $<0.05$ \\
\hline standing long jump (cm) & 102 & 221 & 83 & 324 & 19 & 4 & $(11 ; 27)$ & 0.82 & (moderate) & 4.657 & $<0.01$ \\
\hline 25-m dash $(\mathrm{s})$ & 5.89 & 0.74 & 6.31 & 0.76 & 0.42 & 0.13 & $(0.68 ; 0.15)$ & 0.55 & (small) & 3.132 & $<0.05$ \\
\hline 105 -m agility $(\mathrm{s})^{*}$ & 25.42 & 2.43 & 27.35 & 2.43 & 1.93 & 0.43 & $(2.78 ; 1.08)$ & 0.79 & (moderate) & 4.489 & $<0.01$ \\
\hline 20-m shuttle run $(m)^{*}$ & 295 & 169 & 269 & 125 & 256 & 26 & $(26 ; 77)$ & 0.17 & (trivial) & 0.976 & 0.331 \\
\hline Sit-and-reach $(\mathrm{cm})$ & 24.9 & 5.7 & 26.1 & 6.0 & 1.2 & 1.0 & $(3.3 ; 0.8)$ & 0.21 & (small) & 1.161 & 0.248 \\
\hline Walking backward (\#) & 37.0 & 14.7 & 39.3 & 12.4 & 2.3 & 2.4 & $(7.0 ; 2.5)$ & 0.17 & (trivial) & 0.935 & 0.351 \\
\hline Jumping sideways (\#)* & 32.3 & 9.4 & 30.4 & 9.4 & 1.9 & 1.7 & $(1.4 ; 5.2)$ & 0.20 & (small) & 1.139 & 0.257 \\
\hline Moving sideways (\#)* & 32.1 & 6.8 & 31.9 & 8.3 & 0.2 & 1.3 & $(2.5 ; 2.8)$ & 0.02 & (trivial) & 0.117 & 0.907 \\
\hline Hoping for height (\#) & 35.8 & 11.9 & 30.4 & 12.4 & 5.4 & 2.2 & $(1.1 ; 9.6)$ & 0.44 & (small) & 2.498 & $<0.05$ \\
\hline
\end{tabular}

* test of equality of means was performed on log-transformed variable; SE (standard error); 95\%CL (95\% confidence limits); d (d-Cohen for determining effect size); PMS (predicted mature stature); \# (no measurement unit). 
Table 3. Coefficient correlations among somatic maturation (left: given by attained predicted mature stature expressed in \%; right: given by z-score values obtained using Berkeley Guidance Study) and anthropometry, performance and motor coordination for the total sample and, separately, for boys and girls.

\begin{tabular}{|c|c|c|c|c|c|c|c|c|c|}
\hline \multirow[b]{3}{*}{ Group } & \multirow[b]{3}{*}{$\mathrm{Y}_{\mathrm{i}}$ : Variables } & \multicolumn{4}{|c|}{ X1: Attained predicted mature stature (\%) } & \multicolumn{4}{|c|}{$\mathrm{X} 2$ : Attained predicted mature stature (z-score) } \\
\hline & & \multirow[b]{2}{*}{$\mathrm{r}$} & \multicolumn{2}{|c|}{$95 \% \mathrm{CL}$} & \multirow[b]{2}{*}{$\mathrm{p}$} & \multirow[b]{2}{*}{$\mathrm{r}$} & \multicolumn{2}{|c|}{$95 \% \mathrm{CL}$} & \multirow[b]{2}{*}{$\mathrm{p}$} \\
\hline & & & LL & UL & & & LL & UL & \\
\hline \multirow{17}{*}{$\begin{array}{l}\text { Total } \\
\text { (n } 1 / 4128)\end{array}$} & Stature & 0.260 & 0.091 & 0.429 & $<0.05$ & 0.354 & 0.191 & 0.517 & $<0.01$ \\
\hline & Log (body mass) & 0.377 & 0.215 & 0.539 & $<0.01$ & 0.673 & 0.544 & 0.802 & $<0.01$ \\
\hline & $\log (\mathrm{FM}-\%)$ & 0.513 & 0.363 & 0.663 & $<0.01$ & 0.475 & 0.321 & 0.629 & $<0.01$ \\
\hline & $\log (\mathrm{FM}-\mathrm{kg})$ & 0.484 & 0.331 & 0.637 & $<0.01$ & 0.589 & 0.448 & 0.730 & $<0.01$ \\
\hline & FFM & 0.207 & 0.036 & 0.378 & $<0.05$ & 0.535 & 0.387 & 0.683 & $<0.01$ \\
\hline & 2-kg ball throw & 0.070 & 0.244 & 0.104 & 0.43 & 0.332 & 0.167 & 0.497 & $<0.01$ \\
\hline & Log (hand grip strength) & 0.052 & 0.123 & 0.226 & 0.56 & 0.380 & 0.218 & 0.542 & $<0.01$ \\
\hline & 60-s sit-ups & 0.294 & 0.461 & 0.127 & $<0.05$ & 0.151 & 0.324 & 0.022 & 0.089 \\
\hline & Standing long jump & 0.359 & 0.522 & 0.196 & $<0.01$ & 0.104 & 0.277 & 0.070 & 0.25 \\
\hline & 25-m dash & 0.157 & 0.329 & 0.016 & 0.08 & 0.048 & 0.126 & 0.223 & 0.59 \\
\hline & $\log (10 \quad 5-m$ agility $)$ & 0.357 & 0.520 & 0.194 & $<0.01$ & 0.030 & 0.144 & 0.205 & 0.74 \\
\hline & $\log (20-m$ shuttle run) & 0.233 & 0.403 & 0.063 & $<0.05$ & 0.290 & 0.457 & 0.123 & $<0.05$ \\
\hline & Sit-and-reach & 0.038 & 0.137 & 0.212 & 0.67 & 0.014 & 0.160 & 0.189 & 0.87 \\
\hline & Walking backward & 0.064 & 0.239 & 0.110 & 0.47 & 0.347 & 0.511 & 0.183 & $<0.01$ \\
\hline & Log(Jumping sidways) & 0.168 & 0.340 & 0.004 & 0.06 & 0.163 & 0.336 & 0.009 & 0.07 \\
\hline & Log (moving sideways) & 0.113 & 0.286 & 0.061 & 0.20 & 0.142 & 0.315 & 0.031 & 0.11 \\
\hline & Hoping for height & 0.267 & 0.435 & 0.099 & $<0.05$ & 0.146 & 0.319 & 0.027 & 0.10 \\
\hline
\end{tabular}


Table 3. Continued

\begin{tabular}{|c|c|c|c|c|c|c|c|c|c|}
\hline \multirow[b]{3}{*}{ Group } & \multirow[b]{3}{*}{$\mathrm{Y}_{\mathrm{i}}$ : Variables } & \multicolumn{4}{|c|}{ X1: Attained predicted mature stature $(\%)$} & \multicolumn{4}{|c|}{ X2: Attained predicted mature stature (z-score) } \\
\hline & & \multirow[b]{2}{*}{$\mathrm{r}$} & \multicolumn{2}{|c|}{$95 \% \mathrm{CL}$} & \multirow[b]{2}{*}{$\mathrm{p}$} & \multirow[b]{2}{*}{$\mathrm{r}$} & \multicolumn{2}{|c|}{$95 \% \mathrm{CL}$} & \multirow[b]{2}{*}{$\mathrm{p}$} \\
\hline & & & LL & UL & & & LL & $\mathrm{UL}$ & \\
\hline \multirow{17}{*}{$\begin{array}{l}\text { Girls } \\
\left(n^{1 / 4} 67\right)\end{array}$} & Stature & 0.570 & 0.370 & 0.770 & $<0.01$ & 0.379 & 0.154 & 0.604 & $<0.05$ \\
\hline & Log (body mass) & 0.909 & 0.808 & 1.010 & $<0.01$ & 0.755 & 0.596 & 0.914 & $<0.01$ \\
\hline & $\log (\mathrm{FM}-\%)$ & 0.693 & 0.518 & 0.868 & $<0.01$ & 0.653 & 0.469 & 0.837 & $<0.01$ \\
\hline & $\log (\mathrm{FM}-\mathrm{kg})$ & 0.838 & 0.705 & 0.971 & $<0.01$ & 0.737 & 0.573 & 0.901 & $<0.01$ \\
\hline & FFM & 0.881 & 0.766 & 0.996 & $<0.01$ & 0.708 & 0.536 & 0.880 & $<0.01$ \\
\hline & 2-kg ball throw & 0.480 & 0.267 & 0.693 & $<0.01$ & 0.365 & 0.139 & 0.591 & $<0.05$ \\
\hline & Log (hand grip strength) & 0.511 & 0.302 & 0.720 & $<0.01$ & 0.431 & 0.212 & 0.650 & $<0.01$ \\
\hline & 60 -s sit-ups & 0.214 & 0.451 & 0.024 & 0.08 & 0.170 & 0.410 & 0.069 & 0.17 \\
\hline & Standing long jump & 0.108 & 0.350 & 0.134 & 0.38 & 0.173 & 0.413 & 0.066 & 0.16 \\
\hline & 25-m dash & 0.164 & 0.076 & 0.404 & 0.19 & 0.085 & 0.157 & 0.327 & 0.49 \\
\hline & $\log (10 \quad 5-m$ agility $)$ & 0.019 & 0.262 & 0.224 & 0.88 & 0.113 & 0.355 & 0.128 & 0.36 \\
\hline & Log (20-m shuttle run) & 0.297 & 0.529 & 0.065 & $<0.05$ & 0.282 & 0.515 & 0.049 & $<0.05$ \\
\hline & Sit-and-reach & 0.160 & 0.400 & 0.080 & 0.20 & 0.175 & 0.414 & 0.064 & 0.16 \\
\hline & Walking backward & 0.310 & 0.541 & 0.079 & $<0.05$ & 0.283 & 0.516 & 0.050 & $<0.05$ \\
\hline & Log(Jumping sidways) & 0.096 & 0.338 & 0.146 & 0.44 & 0.168 & 0.408 & 0.072 & 0.17 \\
\hline & Log (moving sideways) & 0.241 & 0.477 & 0.005 & $<0.05$ & 0.165 & 0.404 & 0.075 & 0.18 \\
\hline & Hoping for height & 0.209 & 0.446 & 0.029 & 0.09 & 0.227 & 0.464 & 0.010 & 0.07 \\
\hline
\end{tabular}


Table 3. Continued

\begin{tabular}{|c|c|c|c|c|c|c|c|c|c|}
\hline \multirow[b]{3}{*}{ Group } & \multirow[b]{3}{*}{$\mathrm{Y}_{\mathrm{i}}$ : Variables } & \multicolumn{4}{|c|}{$\mathrm{X} 1$ : Attained predicted mature stature $(\%)$} & \multicolumn{4}{|c|}{$\mathrm{X} 2$ : Attained predicted mature stature (z-score) } \\
\hline & & \multirow[b]{2}{*}{$\mathrm{r}$} & \multicolumn{2}{|c|}{$95 \% \mathrm{CL}$} & \multirow[b]{2}{*}{$\mathrm{p}$} & \multirow[b]{2}{*}{$\mathrm{r}$} & \multicolumn{2}{|c|}{$95 \% \mathrm{CL}$} & \multirow[b]{2}{*}{$\mathrm{p}$} \\
\hline & & & LL & UL & & & LL & UL & \\
\hline Boys & Stature & 0.634 & 0.437 & 0.831 & $<0.01$ & 0.281 & 0.036 & 0.526 & $<0.05$ \\
\hline \multirow[t]{16}{*}{$\left(\mathrm{n}^{1 / 4} 61\right)$} & Log (body mass) & 0.729 & 0.554 & 0.904 & $<0.01$ & 0.565 & 0.354 & 0.776 & $<0.01$ \\
\hline & $\log (\mathrm{FM}-\%)$ & 0.591 & 0.385 & 0.797 & $<0.01$ & 0.568 & 0.358 & 0.778 & $<0.01$ \\
\hline & Log (FM-kg) & 0.660 & 0.468 & 0.852 & $<0.01$ & 0.583 & 0.376 & 0.790 & $<0.01$ \\
\hline & FFM & 0.604 & 0.401 & 0.807 & $<0.01$ & 0.190 & 0.060 & 0.441 & 0.14 \\
\hline & 2-kg ball throw & 0.408 & 0.175 & 0.641 & $<0.05$ & 0.168 & 0.084 & 0.419 & 0.20 \\
\hline & Log (hand grip strength) & 0.413 & 0.181 & 0.645 & $<0.05$ & 0.295 & 0.051 & 0.539 & $<0.05$ \\
\hline & 60 -s sit-ups & 0.297 & 0.541 & 0.053 & $<0.05$ & 0.255 & 0.502 & 0.008 & $<0.05$ \\
\hline & Standing long jump & 0.073 & 0.328 & 0.181 & 0.58 & 0.286 & 0.531 & 0.041 & $<0.05$ \\
\hline & 25-m dash & 0.009 & 0.264 & 0.247 & 0.95 & 0.154 & 0.407 & 0.098 & 0.24 \\
\hline & $\log (10 \quad 5-m$ agility $)$ & 0.215 & 0.464 & 0.034 & 0.096 & 0.015 & 0.270 & 0.240 & 0.91 \\
\hline & Log (20-m shuttle run) & 0.466 & 0.692 & 0.240 & $<0.01$ & 0.343 & 0.583 & 0.103 & $<0.05$ \\
\hline & Sit-and-reach & 0.076 & 0.179 & 0.330 & 0.56 & 0.339 & 0.099 & 0.579 & $<0.05$ \\
\hline & Walking backward & 0.108 & 0.362 & 0.145 & 0.41 & 0.411 & 0.644 & 0.178 & $<0.05$ \\
\hline & Log(Jumping sidways) & 0.220 & 0.469 & 0.029 & 0.09 & 0.238 & 0.486 & 0.010 & 0.07 \\
\hline & Log (moving sideways) & 0.000 & 0.255 & 0.255 & 1.00 & 0.134 & 0.387 & 0.119 & 0.30 \\
\hline & Hoping for height & 0.080 & 0.334 & 0.175 & 0.54 & 0.185 & 0.436 & 0.066 & 0.15 \\
\hline
\end{tabular}

95\% CL (95\% confidence limits); LL (lower limit); UL (upper limit); FM-\% (fat mass percentage); FM-kg (fat mass); FFM (fat free mass). 
Table 4. Means and standard deviations for girls contrasting in somatic maturation given by z-score of attained predicted mature stature (low z scores: $\mathrm{P}<50 \%$; high $\mathrm{z}$ scores: $\mathrm{P}>50 \%$ ) including mean differences magnitude effect and results of $\mathrm{t}$ test for comparisons of means.

\begin{tabular}{|c|c|c|c|c|c|c|c|c|c|}
\hline \multirow[b]{3}{*}{$\underline{Y}_{i}:$ Independent variables } & \multicolumn{4}{|c|}{$\mathrm{X}$ : Contrasting somatic maturation } & \multirow{3}{*}{$\begin{array}{c}\text { Mean differences } \\
(95 \% \mathrm{CL})\end{array}$} & \multicolumn{2}{|c|}{ Magnitude effect } & \multicolumn{2}{|c|}{ Student's t-test } \\
\hline & Low & & high z-scores & $\left(\mathrm{n}^{1 / 4} 33\right)$ & & d & (Qualitative) & $\mathrm{t}$ & $\mathrm{p}$ \\
\hline & Z-scores & $\left(\mathrm{n}^{1 / 4} \underline{33)}\right.$ & & & & & & & \\
\hline $\mathrm{Y}_{1}:$ Chronological age (years) & 8.6 & 0.3 & 8.3 & 0.3 & $0.3(0.1 ; 0.4)$ & 0.97 & (moderate) & 3.950 & $<0.01$ \\
\hline $\mathrm{Y}_{2}:$ Stature $(\mathrm{cm})$ & 128.9 & 5.4 & 132.5 & 6.1 & $3.6(6.4 ; 7.5)$ & 0.62 & (moderate) & 2.528 & $<0.05$ \\
\hline $\mathrm{Y}_{3}:$ Body mass (kg) & 26.4 & 4.4 & 34.5 & 6.3 & $8.1(10.8 ; 5.5)$ & 1.49 & (large) & 6.098 & $<0.01$ \\
\hline $\mathrm{Y}_{4}:$ Fat mass $(\%)$ & 25.0 & 4.5 & 31.5 & 6.0 & $6.5(9.1 ; 3.9)$ & 1.22 & (large) & 5.004 & $<0.01$ \\
\hline $\mathrm{Y}_{5}:$ Fat mass $(\mathrm{kg})$ & 6.7 & 2.2 & 11.2 & 4.2 & $4.5(6.1 ; \quad 2.8)$ & 1.30 & (large) & 5.339 & $<0.01$ \\
\hline $\mathrm{Y}_{6}:$ Fat-free mass $(\mathrm{kg})$ & 19.7 & 2.5 & 23.4 & 2.8 & $3.6(5.0 ; \quad 2.4)$ & 1.40 & (large) & 5.744 & $<0.01$ \\
\hline$Y_{7}: 2-k g$ ball throw $(\mathrm{cm})$ & 169 & 24 & 185 & 32 & $17(7 ; 30)$ & 0.58 & (small) & 2.393 & $<0.05$ \\
\hline $\mathrm{Y}_{8}:$ Hand grip strength (kg.f) & 11.2 & 2.4 & 13.4 & 2.8 & $2.2(3.4 ; 0.9)$ & 0.82 & (moderate) & 3.365 & $<0.05$ \\
\hline $\mathrm{Y}_{9}:$ 60-s sit-ups (\#) & 15.5 & 7.3 & 14.4 & 10.4 & $0.9(3.4 ; 5.4)$ & 0.11 & (trivial) & 0.461 & 0.646 \\
\hline $\mathrm{Y}_{10}:$ Standing long jump $(\mathrm{cm})$ & 88 & 27 & 79 & 21 & $9(3 ; 21)$ & 0.38 & (small) & 1.560 & 0.124 \\
\hline $\mathrm{Y}_{11}: 25-\mathrm{m}$ dash $(\mathrm{s})$ & 6.42 & 0.62 & 6.20 & 0.87 & $0.22(0.15 ; 0.59)$ & 0.29 & (small) & 1.171 & 0.246 \\
\hline $\mathrm{Y}_{12}: 10$ 5-m agility $(\mathrm{s})$ & 27.23 & 2.64 & 27.47 & 2.24 & $0.24(1.43 ; 0.96)$ & 0.10 & (trivial) & 0.394 & 0.695 \\
\hline $\mathrm{Y}_{13}: 20-\mathrm{m}$ shuttle run $(\mathrm{s})$ & 287 & 100 & 252 & 144 & $34(27 ; 95)$ & 0.28 & (small) & 1.127 & 0.264 \\
\hline $\mathrm{Y}_{14:}:$ Sit-and-reach $(\mathrm{cm})$ & 26.8 & 6.3 & 25.5 & 5.7 & $1.3(1.6 ; 4.3)$ & 0.22 & (small) & 0.897 & 0.373 \\
\hline $\mathrm{Y}_{15}:$ Walking backward (\#) & 41.9 & 12.6 & 36.8 & 11.7 & $5.1(0.8 ; 11.1)$ & 0.42 & (small) & 1.718 & 0.090 \\
\hline Y $_{16}$ : Jumping sideways (\#) & 31.7 & 10.9 & 29.2 & 7.7 & $2.5(2.1 ; 7.1)$ & 0.26 & (small) & 1.082 & 0.283 \\
\hline Y $_{17}$ : Moving sideways (\#) & 32.9 & 9.9 & 31.0 & 6.3 & $1.9(2.2 ; 6.0)$ & 0.23 & (small) & 0.946 & 0.348 \\
\hline$\underline{Y}_{18}:$ Hoping for height (\#) & 32.7 & -12.6 & 28.2 & -12.0 & $4.5(1.5 ; 10.5)$ & 0.37 & (trivial) & 1.501 & 0.138 \\
\hline
\end{tabular}

95\%CL (95\% confidence limits); \# (no measurement unit). 
Table 5. Means and standard deviations for boys contrasting in somatic maturation given by $\mathrm{z}$ score of attained predicted mature stature (low z scores: $\mathrm{P}<50 \%$; high z scores: $\mathrm{P}>50 \%$ ) including mean differences magnitude effect and results of $\mathrm{t}$ test for comparisons of means.

\begin{tabular}{|c|c|c|c|c|c|c|c|c|c|}
\hline \multirow[b]{3}{*}{$\underline{Y}_{\mathrm{i}}:$ independent variable } & \multicolumn{4}{|c|}{ Contrasting somatic maturation } & \multirow[b]{3}{*}{ Mean differences (95\% CL) } & \multicolumn{2}{|c|}{ Magnitude effect } & \multicolumn{2}{|c|}{ Student's t-test } \\
\hline & \multirow{2}{*}{\multicolumn{2}{|c|}{$\begin{array}{l}\text { Low z-scores } \\
\left(\mathrm{n}^{1 / 4} \underline{33)}\right.\end{array}$}} & \multirow{2}{*}{\multicolumn{2}{|c|}{$\begin{array}{l}\text { High z-scores } \\
(\mathrm{n} 1 / 4 \underline{33)}\end{array}$}} & & $\mathrm{d}$ & (Qualitative) & $\mathrm{t}$ & $\mathrm{p}$ \\
\hline & & & & & & & & & \\
\hline $\mathrm{Y}_{1}$ : Chronological age (years) & 8.6 & 0.3 & 8.4 & 0.3 & $0.2(0.1 ; 0.4)$ & 0.83 & (moderate) & 3.222 & $<0.05$ \\
\hline $\mathrm{Y}_{2}:$ Stature $(\mathrm{cm})$ & 131.2 & 6.1 & 132.9 & 4.7 & $1.7(4.5 ; 1.1)$ & 0.31 & (small) & 1.201 & 0.235 \\
\hline $\mathrm{Y}_{3}$ : Body mass (kg) & 29.5 & 5.3 & 35.6 & 9.1 & $6.1(9.9 ; \quad 2.2)$ & 0.81 & (moderate) & 3.152 & $<0.05$ \\
\hline $\mathrm{Y}_{4}:$ Fat mass $(\%)$ & 21.9 & 7.4 & 29.6 & 11.7 & $7.7(\quad 12.7 ; \quad 2.6)$ & 0.79 & (moderate) & 3.048 & $<0.05$ \\
\hline $\mathrm{Y}_{5}:$ Fat mass $(\mathrm{kg})$ & 6.8 & 3.4 & 11.5 & 7.9 & $4.7(7.8 ; 1.5)$ & 0.77 & (moderate) & 2.985 & $<0.05$ \\
\hline $\mathrm{Y}_{6}$ : Fat-free mass $(\mathrm{kg})$ & 22.8 & 2.8 & 24.1 & 2.8 & $1.3(2.8 ; 0.1)$ & 0.49 & (small) & 1.895 & 0.063 \\
\hline $\mathrm{Y}_{7}:$ 2-kg ball throw $(\mathrm{cm})$ & 197 & 30 & 210 & 38 & $12(30 ; 5)$ & 0.37 & (small) & 1.419 & 0.161 \\
\hline $\mathrm{Y}_{8}:$ Hand grip strength (kg.f) & 14.5 & 5.0 & 15.0 & 3.6 & $0.5(2.8 ; 1.7)$ & 0.13 & (trivial) & 0.492 & 0.624 \\
\hline $\mathrm{Y}_{9}: 60-\mathrm{s}$ sit-ups (\#) & 19.7 & 8.3 & 17.4 & 10.6 & $2.3(2.6 ; 7.2)$ & 0.24 & (small) & 0.934 & 0.354 \\
\hline $\mathrm{Y}_{10}:$ Standing long jump $(\mathrm{cm})$ & 110 & 19 & 95 & 21 & $15(5 ; 25)$ & 0.76 & (moderate) & 2.938 & $<0.05$ \\
\hline $\mathrm{Y}_{11}: 25-\mathrm{m}$ dash $(\mathrm{s})$ & 5.84 & 0.62 & 5.94 & 0.85 & $0.10(0.49 ; 0.3)$ & 0.14 & (trivial) & 0.539 & 0.592 \\
\hline $\mathrm{Y}_{12}: 10$ 5-m agility (s) & 25.31 & 1.98 & 25.53 & 2.82 & $0.22(1.48 ; 1.0)$ & 0.09 & (trivial) & 0.361 & 0.719 \\
\hline $\mathrm{Y}_{13}: 20-\mathrm{m}$ shuttle run $(\mathrm{s})$ & 306 & 123 & 284 & 206 & $22(65 ; 109)$ & 0.13 & (trivial) & 0.507 & 0.614 \\
\hline $\mathrm{Y}_{14:}$ Sit-and-reach $(\mathrm{cm})$ & 23.9 & 5.9 & 25.9 & 5.6 & $2.0(4.9 ; 1.0)$ & 0.34 & (small) & 1.335 & 0.187 \\
\hline $\mathrm{Y}_{15}$ : Walking backward (\#) & 42.8 & 14.4 & 31.5 & 12.8 & $11.3(4.3 ; 18.3)$ & 0.84 & (moderate) & 3.246 & $<0.05$ \\
\hline $\mathrm{Y}_{16}:$ Jumping sideways (\#) & 34.5 & 9.1 & 30.2 & 9.3 & $4.3(0.4 ; 9.0)$ & 0.47 & (small) & 1.815 & 0.075 \\
\hline $\mathrm{Y}_{17}:$ Moving sideways (\#) & 34.0 & 7.1 & 30.3 & 6.2 & $3.7(0.4 ; 7.1)$ & 0.57 & (small) & 2.205 & $<0.05$ \\
\hline$\underline{Y}_{18}$ : Hoping for height (\#) & 39.3 & -9.8 & 32.5 & -12.9 & $6.8(0.9 ; 12.7)$ & 0.60 & (moderate) & 2.305 & $<0.05$ \\
\hline
\end{tabular}

95\%CL (95\% confidence limits); \# (no measurement unit). 
Table 6. Factorial multivariate analyses of variance (factorial MANOVA) to examine the effects of sex, contrasting maturation and interaction term on fitness and motor coordination; and results of multivariate analyses of covariance (MANCOVA with somatic maturation given by z-scores of attained predicted mature stature as covariate) to examine the effects of sex on fitness and motor coordination.

\begin{tabular}{|c|c|c|c|c|c|c|c|c|c|c|c|c|c|}
\hline \multirow{2}{*}{$\begin{array}{l}\mathrm{Y}_{\mathrm{i}} \text { : Dependent } \\
\text { variables }\end{array}$} & \multirow[b]{2}{*}{ Test } & \multicolumn{4}{|c|}{$\mathrm{X}_{1}: \operatorname{Sex}$} & \multicolumn{4}{|c|}{$\begin{array}{l}\mathrm{X}_{2} \text { : Maturity } \\
\text { status }^{\mathrm{a}}\end{array}$} & \multicolumn{4}{|c|}{ Interaction $\left(\mathrm{X}_{1} . \mathrm{X}_{2}\right)$} \\
\hline & & Wilks' & $\mathrm{F}$ & $\mathrm{p}$ & ES-r & Wilks' & $\mathrm{F}$ & $\mathrm{p}$ & ES-r & Wilks' & $\mathrm{F}$ & $\mathrm{p}$ & ES-r \\
\hline Y: Fitness & MANOVA & 0.701 & 6.250 & $<0.05$ & & 0.822 & 3.165 & $<0.05$ & & 0.960 & 0.604 & 0.773 & \\
\hline $\mathrm{Y}_{1}:$ 2-kg ball throw & ANOVA & & 23.454 & $<0.05$ & 0.40 & & 6.851 & $<0.05$ & 0.23 & & 0.132 & 0.717 & 0.03 \\
\hline $\mathrm{Y}_{2}: \log$ (hand grip) & ANOVA & & 8.525 & $<0.05$ & 0.25 & & 6.645 & $<0.05$ & 0.23 & & 0.717 & 0.399 & 0.08 \\
\hline $\mathrm{Y}_{3}: 60-\mathrm{s}$ sit-ups & ANOVA & & 4.779 & $<0.05$ & 0.19 & & 1.011 & 0.317 & 0.09 & & 0.150 & 0.700 & 0.03 \\
\hline $\mathrm{Y}_{4}:$ Standing long jump & ANOVA & & 23.149 & $<0.05$ & 0.40 & & 9.428 & $<0.05$ & 0.27 & & 0.529 & 0.468 & 0.07 \\
\hline $\mathrm{Y}_{5}: 25-\mathrm{m}$ dash & ANOVA & & 9.898 & $<0.05$ & 0.27 & & 0.180 & 0.672 & 0.04 & & 1.440 & 0.232 & 0.11 \\
\hline $\mathrm{Y}_{6}: \log (10 \quad 5-\mathrm{m}$ agility $)$ & ANOVA & & 21.254 & $<0.05$ & 0.38 & & 0.249 & 0.619 & 0.04 & & 0.013 & 0.908 & 0.01 \\
\hline$Y_{7}: \log (20-m$ shuttle run $)$ & ANOVA & & 0.100 & 0.752 & 0.03 & & 4.675 & $<0.05$ & 0.19 & & 0.033 & 0.857 & 0.02 \\
\hline $\mathrm{Y}_{8}:$ Sit-and-reach & ANOVA & & 1.411 & 0.237 & 0.11 & & 0.094 & 0.760 & 0.03 & & 2.479 & 0.118 & 0.14 \\
\hline Z: Coordination & MANOVA & 0.907 & 3.118 & $<0.05$ & & 0.878 & 4.186 & $<0.05$ & & 0.981 & 0.575 & 0.681 & \\
\hline $\mathrm{Z}_{1}$ : Walking backward & ANOVA & & 0.914 & 0.341 & 0.09 & & 12.985 & $<0.05$ & 0.31 & & 1.850 & 0.176 & 0.12 \\
\hline $\mathrm{Z}_{2}$ : Log (jumping sideways) & ANOVA & & 1.531 & 0.218 & 0.11 & & 2.689 & 0.104 & 0.15 & & 0.837 & 0.362 & 0.08 \\
\hline $\mathrm{Z}_{3}: \log$ (moving sideways) & ANOVA & & 0.085 & 0.772 & 0.03 & & 4.676 & $<0.05$ & 0.19 & & 0.765 & 0.383 & 0.08 \\
\hline $\mathrm{Z}_{4}:$ Hoping for height & ANOVA & & 6.556 & $<0.05$ & 0.22 & & 7.160 & $<0.05$ & 0.23 & & 0.287 & 0.593 & 0.05 \\
\hline Y': Fitness & MANCOVA & 0.719 & 5.761 & $<0.05$ & & & & & & & & & \\
\hline $\mathrm{Y}_{1}^{\prime}: 2-\mathrm{kg}$ ball throw & ANCOVA & & 16.168 & $<0.05$ & 0.34 & & & & & & & & \\
\hline $\mathrm{Y}_{2}{ }_{2}: \log$ (hand grip) & ANCOVA & & 3.840 & 0.052 & 0.17 & & & & & & & & \\
\hline $\mathrm{Y}_{3}^{\prime}: 60$-s sit-ups & ANCOVA & & 7.427 & $<0.05$ & 0.24 & & & & & & & & \\
\hline$Y_{4}^{\prime}:$ Standing long jump & ANCOVA & & 27.143 & $<0.05$ & 0.42 & & & & & & & & \\
\hline
\end{tabular}


Table 6. Continued

\begin{tabular}{|c|c|c|c|c|c|c|c|c|c|c|c|c|c|}
\hline \multirow{2}{*}{$\begin{array}{l}\mathrm{Y}_{\mathrm{i}} \text { : Dependent } \\
\text { variables }\end{array}$} & \multirow[b]{2}{*}{ Test } & \multicolumn{4}{|c|}{$\mathrm{X}_{1}:$ Sex } & \multicolumn{4}{|c|}{$\begin{array}{l}\mathrm{X}_{2} \text { : Maturity } \\
\text { status }^{\mathrm{a}}\end{array}$} & \multicolumn{4}{|c|}{ Interaction $\left(\mathrm{X}_{1} . \mathrm{X}_{2}\right)$} \\
\hline & & Wilks' & $\mathrm{F}$ & $\mathrm{p}$ & ES-r & Wilks' & $\mathrm{F}$ & $\mathrm{p}$ & ES-r & Wilks' & $\mathrm{F}$ & $\mathrm{p}$ & ES-r \\
\hline $\mathrm{Y}_{5}{ }_{5}: 25-\mathrm{m}$ dash & ANCOVA & & 9.454 & $<0.05$ & 0.27 & & & & & & & & \\
\hline$Y_{6}^{\prime}: \log (10 \quad 5-m$ agility $)$ & ANCOVA & & 21.948 & $<0.05$ & 0.39 & & & & & & & & \\
\hline$Y_{7}^{\prime}: \log (20-m$ shuttle run) & ANCOVA & & 1.368 & 0.244 & 0.10 & & & & & & & & \\
\hline$Y_{8}^{\prime}:$ Sit-and-reach & ANCOVA & & 1.516 & 0.221 & 0.11 & & & & & & & & \\
\hline Z': Coordination & MANCOVA & 0.911 & 2.963 & $<0.05$ & & & & & & & & & \\
\hline $\mathrm{Z}_{1}^{\prime}$ : Walking backward & ANCOVA & & 0.000 & 0.999 & $<0.01$ & & & & & & & & \\
\hline $\begin{array}{l}Z_{2}^{\prime}: \text { Log (jumping } \\
\text { sideways) }\end{array}$ & ANCOVA & & 3.000 & 0.086 & 0.15 & & & & & & & & \\
\hline $\mathrm{Z}_{3}^{\prime}:$ Log (moving sideways) & ANCOVA & & 0.458 & 0.500 & 0.06 & & & & & & & & \\
\hline$Z_{4}^{\prime}:$ Hoping for height & ANCOVA & & 9.249 & $<0.05$ & 0.26 & & & & & & & & \\
\hline
\end{tabular}

${ }^{\mathrm{a}}$ (contrasting maturity groups obtained from earliest and latest values of z-score of attained predicted mature stature using means and standard deviations of the Berkeley Guidance Study). 
Means and standard deviations for girls contrasting somatic maturation given by $\mathrm{z}$ score of attained percentage of mature stature (low z scores: $\mathrm{P}$ $<50 \%$; high $\mathrm{z}$ scores: $\mathrm{P}>50 \%$ ) are presented in Table 4, which also includes differences between both $\mathrm{z}$ scores groups. Girls contrasting in maturation differed significantly for body mass, estimated fat mass and estimated fat-free mass, 2-kg BT, and HGP.

Descriptive statistics for boys contrasting in maturation given by $\mathrm{z}$ score of attained percentage of mature stature (low $\mathrm{z}$ scores: $\mathrm{P}<50 \%$; high $\mathrm{Z}$ scores: $\mathrm{P}>50 \%$ ) are presented in Table 5. Differences were significant for body mass, estimated fat mass, and SLJ. Among the four items from the KTK battery, boys contrasting in maturation differed on $\mathrm{WB}, \mathrm{MS}$, and $\mathrm{HH}$.

Table 6 summarizes the MANOVA for the eight physical fitness tests and showed a significant sex effect and maturity status effect. In addition, subsequent ANOVAs were produced separately for each test, and boys performed better scores compared with girls. Differences were significant for 2-kg medicine BT, HGP, 60-s SUP, SLJ, 25-m dash, and 10 5-m agility. No significant difference was found for the interaction sex maturation on physical fitness tests. The MANOVA for the four KTK items showed a significant effect for sex and maturity status. Subsequent ANOVAs presented boys having higher mean values than girls in the HH. The interaction term sex maturity status was not significant for motor coordination items. The results of the MANCOVA to examine sex differences (with maturation as covariate) are presented in Table 6. The MANCOVA showed a significant effect for sex on all physical fitness tests and for all KTK items. For the physical fitness domain, significant differences were found, with boys attaining better scores on 2-kg medicine BT, 60-s SUP, SLJ, 25-m dash, and 10 5$m$ agility.

Finally, the KTK indicated boys had significantly better scores than girls in the protocol $\mathrm{HH}$.

\section{Discussion}

The results of the current investigation demonstrate that boys performed better than girls on all but two of the fitness tests (the $20-\mathrm{m} \mathrm{SHR}$ and the SAR tests) and all of the KTK tests of motor competence, with the exception of $\mathrm{HH}$. The results also suggested that sex-related differences that were not substantially explained by biological maturation given by $\mathrm{z}$ scores of attained PMS. The analyses performed in the current study did not control for inter-individual variation in chronological 
age because inclusion criteria considered age limits of 8.0 to 8.9 years. Consequently, the comparisons between boys and girls were repeated after controlling for somatic maturation, and the results remained quite similar. For both sexes, the association between maturation and motor tests (physical fitness and motor coordination) consistently suggested an inverse relationship that was particularly evident on the motor performance items that required body displacement.

Fjørtoft, Pedersen, Sigmundsson, and Vereijken recommended that a battery of physical tests designed to assess motor competence and fitness in young people should include a combination of motor activities including endur- ance, strength, flexibility, agility, and balance. Accordingly, the tests included in the current study assessed a diverse range of motor and functional attributes that are considered to be indicative of physical and functional health in children. Consistent with previous literature (Colella, Morano, Robazza, \& Bortoli, 2009; Drenowatz et al., 2013; Katzmarzyk, Malina, \& Beunen, 1997; Marta, Marinho, Barbosa, Izquierdo, \& Marques, 2012), boys outperformed girls in tests such as static strength, speed, explosive strength, and agility.

Sex differences in fitness and motor competence, and biological maturation, have important implications for engagement in physical activities. Perceptions of physical and sport compe- tence have been documented as important predictors of involvement engage-ment in both physical activity and sport, with children reporting high perceptions of competence being the most active and most likely to participate in sports. Given that boys tend to perform better on tests of motor competence and fitness, it is perhaps not surprising that one of the most consistent findings in pediatric literature is that boys are generally more physically active and less sedentary than girls of the same age (Malina et al., 2004). Whereas sex differ- ences in physical activity and sedentary behavior have traditionally been attrib- uted to variation in education style, social expectations, and other cultural factors, emerging evidence suggests these differences reflect underlying differences in biological maturity (Malina et al., 2004). That is, girls compared to boys of the same chronological age tend to be less active and more sedentary as a consequence of their advanced maturation (Machado Rodrigues et al., 2010; Sherar et al., 2007; Thompson, Baxter-Jones, Mirwald, \& Bailey, 2003).

A number of previous studies have investigated relations between biological maturation, physical fitness, and motor performance in children (Beunen et al., 1997; Drenowatz et al., 2013; Katzmarzyk et al., 1997). In a study of Belgian girls, Beunen et al. (1997) reported an inverse association between skeletal age and performance on a number of health-related physical fitness tests, including bent arm hang, leg lifts, and 
SUP. Similarly, Katzmarzyk et al. (1997) reported that skeletal age was more closely associated with motor fitness (35-yard dash, SLJ, and soft BT for distance tests) than muscular strength (HGP and pushing and pulling strength of the shoulders with a dynamometer tests) in boys and girls aged 7-12 years. Assessing maturity status based on percentage of adult stature attained, Drenowatz et al. (2013) also showed, in a German sample of boys and girls (7.6 0.4 years), that early-maturing children displayed a more adverse cardiovascular risk profile, presented lower physical fitness scores, and spent more time watching TV compared with their peers, consistent with results observed in the present study. Advanced maturation may adversely affect per- formance on a number of tests of both physical fitness and motor competence in Brazilian youth.

It has been suggested that individual differences in maturation status may positively or negatively affect performance on tests of motor competence and physical fitness (Beunen et al., 1997; Drenowatz et al., 2013; Katzmarzyk et al., 1997) and that the nature of the association may vary relative to age and sex of the individual, and the nature and demands of the task. Katzmarzyk et al. (1997) suggested while maturation of the neuromuscular system may contribute posi- tively to the development of motor skill, maturity-related changes in both size and body composition could negatively affect performance, particularly on tasks requiring body displacement, and especially for girls who experience greater pubertal gains in absolute and relative fat mass (Beunen et al., 1997; Drenowatz et al., 2013). That said, higher physical fitness levels in early- maturing children have been shown to be associated with sports participa- tion, even accounting for their increased size and mass (Jones, Hitchen, \& Stratton, 2000).

The development of fundamental motor coordination has received less atten- tion in relation to the processes of growth and maturation (Freitas et al., 2015). Correlations between skeletal age and outcomebased tests for striking, catching, and balance have generally been found to be low to moderate in primary grade children (Seils, 1951), while no significant relationship was reported between the fine motor task and skeletal age in children 5-9 years (Kerr, 1975). Regarding sex differences in gross motor coordination, a study with German children ( $\mathrm{M}$ age 6.7 years) showed that boys scored better than girls in the overall per-formance of KTK (Graf et al., 2004). Lopes et al. (2012) assessed 7,175 Portuguese children aged 6-14 years and reported that boys performed better than girls at all ages for total KTK motor quotient. However, in another recent study with 2,470 Flemish children aged 6-12 years from 26 primary schools, Vandorpe et al. (2011) reported that the raw scores of two KTK items were significantly 
different between boys and girls. Girls attained better scores than boys in $\mathrm{WB}$, while boys performed better on the $\mathrm{HH}$ test.

It is important to note that the potential for sex-related differences in bio- logical maturation to contribute toward sex-related differences in motor com- petence and fitness has not been systematically addressed in the literature. Consequently, it is important to consider and interpret sex differences in fitness and motor competence with caution. In the current study, sex differences were not significant after controlling for somatic maturation, with the exception of hoping for height (boys presented the best values); Vandendriessche et al. (2011) found that maturity status did not load high on motor coordination, which may suggest that somatic maturation does not strongly affect gross motor coordin- ation during the prepubertal years (7-11 years). However, Freitas et al. (2015), in a study conducted with Portuguese children, showed that the results imply a limited role for skeletal maturation per se or interacting with body size in the development of fundamental motor skills and motor coordination among chil- dren 7-10 years of age. In addition, many of the relationships were negative, suggesting that later maturation was associated with better performances on the motor coordination tests.

In summary, the findings of the present study suggest sex-related differences in biological maturation may contribute toward sex-related differences in both physical fitness and motor competence in young children aged 8-9 years. Differences between boys and girls during the first decade of life, and during years of primary education, in particular, may be a consequence of morpho- logical, biological, and cultural factors (Armstrong, Lambert, \& Lambert, 2011; Krombholz, 2006; Malina et al., 2004). The literature emphasizes the importance of motor coordination in prepubertal years, and a link between motor proficiency in early years with physical fitness and physical activity during adolescent and adult years is consistently assumed (Robinson et al., 2015; Stodden et al., 2008). The adequate interpretation of group and individ- ual performances is believed to be crucial for sport and exercise adherence and maintenance in children and youth. Some limitations should be addressed for future research. The cross-sectional design precludes any statements on causality but should be investigated and confirmed by prospective studies. Second, meas- urements of stature, weight, and percentage of body fat were not made at a standardized time for all youth (i.e., during the day and not at the beginning of the day). Finally, this study did not include measurements of physical activity or sports participation. Research conducted with nonathlete children showed that individuals needed a repertoire of gross and fine motor skills to adapt for the demands of school, occupational, and other social contexts (Bouffard, Watkinson, Thompson, Causgrove Dunn, \& 
Romanow, 1996; Losse et al., 1991; Skinner \& Piek, 2001). The findings from the current study are potential contributors for planning of activities that take into account the success and motivation of preteen girls and boys and thus promote subsequent physical activity and physical fitness.

\section{Acknowledgments}

The authors appreciated the comments and suggestions received from Robert Malina (Emeritus Professor at the University of Texas, USA) during the preparation and refine- ment of this article.

\section{Declaration of Conflicting Interests}

The author(s) declared no potential conflicts of interest with respect to the research, authorship, and/or publication of this article.

\section{Funding}

The author(s) disclosed receipt of the following financial support for the research, author- ship, and/or publication of this article: This research was partially supported by CAPES Foundation (BEX 1617/13-3).

\section{References}

Armstrong, M. E., Lambert, E. V., \& Lambert, M. I. (2011). Physical fitness of South African primary school children, 6 to 13 years of age: Discovery vitality health of the nation study. Perceptual and Motor Skills, 113(3), 999-1016.

Baxter-Jones, A. D. G., Eisenmann, J. C., \& Sherar, L. B. (2005). Controlling for matura- tion in pediatric exercise science. Pediatric Exercise Science, 17, 18-30.

Bayer, L. M., \& Bayley, N. (1959). Growth diagnosis: Selected methods for interpreting and predicting development from one year to maturity. Chicago, IL: University of Chicago Press.

Beunen, G. P., Malina, R. M., Lefevre, J., Claessens, A. L., Renson, R., Kanden Eynde, B., .. . Simons, J. (1997). Skeletal maturation, somatic growth and physical fitness in girls 6-16 years of age. International Journal of Sports Medicine, 18(6), 413-419.

Bouffard, M., Watkinson, E. J., Thompson, L. P., Causgrove Dunn, J. L., \& Romanow, S. K. E. (1996). A test of the activity deficit hypothesis with children with movement difficulties. Adapted Physical Activity Quarterly, 13, 61-73.

Burns, Y. R., Danks, M., O'Callaghan, M. J., Gray, P. H., Cooper, D., Poulsen, L., ... Watter, P. (2009). Motor coordination difficulties and physical fitness of extre- mely-low-birthweight children. Developmental Medicine and Child Neurology, 51, 136-142. Cairney, J., Hay, J., Veldhuizen, S., \& Faught, B. (2010). Comparison of $\mathrm{VO} 2$ maximum obtained from $20 \mathrm{~m}$ shuttle run and cycle ergometer in children with and without developmental coordination disorder.Research in Developmental Disabilities, 31, 1332-1339.

Camacho-Araya, T., Woodburn, S. S., \& Boschini, C. (1990). Reliability of the Prueba de Coordinacion Corporal para Ninos (body coordination test for children). Perceptual and Motor Skills, 70(3 Pt 1), 832-834.

Colella, D., Morano, M., Robazza, C., \& Bortoli, L. (2009). Body image, perceived physical ability, and motor performance in nonoverweight and overweight Italian children. Perceptual and Motor Skills, 108(1), 209-218.

Committee for the Development of Sports. (1988). EUROFIT: Handbook for the European test of physical fitness. Rome, Italy: Council of Europe.

Cumming, S. P., Sherar, L. B., Esliger, D. W., Riddoch, C. J., \& Malina, R. M. (2014). Concurrent and prospective associations among biological maturation, and physical activity at 11 and 13 years of age. Scandinavian Journal of Medicine and Science in Sports, 24(1), e20-e28. 
Cumming, S. P., Standage, M., Gillison, F. B., Dompier, T. P., \& Malina, R. M. (2009). Biological maturity status, body size, and exercise behavior in British youth: A pilot study. Journal of Sports Sciences, 27, 677-686.

Cumming, S. P., Standage, M., Gillison, F., \& Malina, R. M. (2008). Sex differences in exercise behavior during adolescence: Is biological maturation a confounding factor? Journal of Adolescent Health, 42, 480-485.

Deprez, D., Valente-dos-Santos, J., Coelho e Silva, M., Lenoir, M., Philippaerts, R. M., \& Vaeyens, R. (2014). Modeling developmental changes in the yo-yo intermittent recovery test level 1 in elite pubertal soccer players. International Journal of Sports Physiology and Performance, 9, 1006-1012.

D'Hondt, E., Gentier, I., Deforche, B., Tanghe, A., De Bourdeaudhuij, I., \& Lenoir, M. (2011). Weight loss and improved gross motor coordination in children as a result of multidisciplinary residential obesity treatment. Obesity (Silver Spring, Md.), 19(10), 1999-2005.

Drenowatz, C., Wartha, O., Klenk, J., Brandstetter, S., Wabitsch, M., \& Steinacker, J. (2013). Differences in health behavior, physical fitness, and cardiovascular risk in early, average, and late mature children. Pediatric Exercise Science, 25(1), 69-83.

Fjørtoft, I., Pedersen, A. V., Sigmundsson, H., \& Vereijken, B. (2011). Measuring phys- ical fitness in children who are 5 to 12 years old with a test battery that is functional and easy to administer. Physical Therapy, 91, 1087-1095.

Freitas, D. L., Lausen, B., Maia, J. A., Lefevre, J., Gouveia, E. R., Thomis, M., Malina, R. M. (2015). Skeletal maturation, fundamental motor skills and motor coordination in children 7-10 years. Journal of Sports Sciences, 33(9), 924-934.

Graf, C., Koch, B., Kretschmann-Kandel, E., Falkowski, G., Christ, H., Coburger, S., ... Dordel, S. (2004). Correlation between BMI, leisure habits and motor abilities in childhood (CHILT-project). International Journal of Obesity and Related Metabolic Disorders?: Journal of the International Association for the Study of Obesity, 28, 22-26.

Graham, D. J., Sirard, J. R., \& Neumark-Sztainer, D. (2011). Adolescents' attitudes toward sports, exercise, and fitness predict physical activity 5 and 10 years later. Preventive Medicine, 52, 130-132.

Hopkins, W. G., Marshall, S. W., Batterham, A. M., \& Hanin, J. (2009). Progressive statistics for studies in sports medicine and exercise science. Medicine and Science in Sports and Exercise, 41(1), 3-13.

Janssen, I., \& Leblanc, A. G. (2010). Systematic review of the health benefits of physical activity and fitness in school-aged children and youth. The International Journal of Behavioral Nutrition and Physical Activity, 7, 40.

Jones, M. A., Hitchen, P. J., \& Stratton, G. (2000). The importance of considering bio- logical maturity when assessing physical fitness measures in girls and boys aged 10 to 16 years. Annals of Human Biology, 27(1), 57-65.

Kakebeeke, T. H., Locatelli, I., Rousson, V., Caflisch, J., \& Jenni, O. G. (2012). Improvement in gross motor performance between 3 and 5 years of age. Perceptual and Motor Skills, 114(3), 795-806.

Katzmarzyk, P. T., Malina, R. M., \& Beunen, G. P. (1997). The contribution of biological maturation to the strength and motor fitness of children. Annals of Human Biology, 24(6), 493-505.

Kerr, R. (1975). Movement control and maturation in elementary-grade children. Perceptual and Motor Skills, 41, 151-154.

Khamis, H. J., \& Roche, A. F. (1994). Predicting adult stature without using skeletal age: The Khamis-Roche method. Pediatrics, 94, 504-507.

Kiphard, E. J., \& Schilling, F. (1974). Ko"r perkoordinationstest für kinder [Body coordination test for children]. Weinheim, Germany: Beltz Test GmbH.

Krombholz, H. (2006). Physical performance in relation to age, sex, birth order, social class, and sports activities of preschool children. Perceptual and Motor Skills, 102(2), 477-484. 
Krombholz, H. (2013). Motor and cognitive performance of overweight preschool chil- dren. Perceptual and Motor Skills, 116, 40-57.

Lohman, T. G., Roche, A. F., \& Martorell, R. (1988). Anthropometric standardization reference manual. Champaign, IL: Human Kinetics.

Lopes, L., Santos, R., Pereira, B., \& Lopes, V. P. (2013). Associations between gross motor coordination and academic achievement in elementary school children. Human Movement Science, 32, 9-20.

Lopes, V. P., Stodden, D. F., Bianchi, M. M., Maia, J. A. R., \& Rodrigues, L. P. (2012). Correlation between BMI and motor coordination in children. Journal of Science and Medicine in Sport, 15, 38-43.

Losse, A., Henderson, S. E., Elliman, D., Hall, D., Knight, E., \& Jongmans, M. J. (1991). Clumsiness in children: Do they grow out of it? A 10-year followup study. Developmental Medicine and Child Neurology, 33, 55-68.

Luz, L. G. O., Seabra, A. F. T., Santos, R., Padez, C., Ferreira, J. P., \& Coelho-eSilva, M. J. (2015). Association between BMI and motor coordination among children (KTK): A meta-analysis. Brazilian journal of Sports Medicine, 21(3), 230-235.

Machado Rodrigues, A. M., Coelho e Silva, M. J., Mota, J., Cumming, S. P., Sherar, L. B., Neville, H., ... Malina, R. M. (2010). Confounding effect of biologic maturation on sex differences in physical activity and sedentary behavior in adolescents. Pediatric Exercise Science, 22(3), 442-453.

Malina, R. M. (2014). Top 10 research questions related to growth and maturation of relevance to physical activity, performance, and fitness. Research Quarterly for Exercise and Sport, 85(2), 157-173.

Malina, R. M., Bouchard, C., \& Bar-Or, O. (2004). Growth, maturation, and physical activity. Champaign, IL: Human Kinetics.

Malina, R. M., Coelho e Silva, M. J., Figueiredo, M. J., Carling, C., \& Beunen, G. P. (2012). Interrelationships among invasive and noninvasive indicators of biological maturation in adolescent male soccer players. Journal of Sports Sciences, 30, 1705-1717.

Malina, R. M., Cumming, S. P., Morano, P. J., Barron, M., \& Miller, S. J. (2005). Maturity status of youth football players: A noninvasive estimate. Medicine and Science in Sports and Exercise, 37, 1044-1052.

Malina, R. M., Dompier, T. P., Powell, J. W., Barron, M. J., \& Moore, M. T. (2007). Validation of a noninvasive maturity estimate relative to skeletal age in youth football players. Clinical Journal of Sports Medicine, 17, 362-368.

Marta, C. C., Marinho, D. A., Barbosa, T. M., Izquierdo, M., \& Marques, M. C. (2012). Physical fitness differences between prepubescent boys and girls. Journal of Strength and Conditioning Research, 26(7), 1756-1766.

Martins, D., Maia, J., Seabra, A., Garganta, R., Lopes, V., Katzmarzyk, P., Beunen, G. (2010). Correlates of changes in BMI of children from the Azores islands. International Journal of Obesity, 34, 1487-1493.

Okely, A. D., Booth, M. L., \& Patterson, J. W. (2001). Relationship of physical activity to fundamental movement skills among adolescents. Medicine and Science in Sports and Exercise, 33, 1899-1904.

Physical Activity Guidelines Advisory Committee. (2008). Physical activity guidelines advisory committee report (Vol. 67, p. 683). Washington, DC: Author. 
Rasmussen, A. R., Wohlfahrt-Veje, C., Tefre de Renzy-Martin, K., Hagen, C. P., Tinggaard, J., Mouritsen, A., ... Main, K. M. (2015). Validity of self-assessment of pubertal maturation. Pediatrics, 135(1), 86-93.

Rivilis, I., Hay, J., Cairney, J., Klentrou, P., Liu, J., \& Faught, B. E. (2011). Physical activity and fitness in children with developmental coordination disorder: A systematic review. Research in Developmental Disabilities, 32(3), 894-910.

Robinson, L. E., Stodden, D. F., Barnett, L. M., Lopes, V. P., Logan, S. W., Rodrigues, L. P., ... D'Hondt, E. (2015). Motor competence and its effect on positive developmental trajectories of health. Sports Medicine, 45(9), 1273-1284.

Seils, L. G. (1951). The relationship between measures of physical growth and gross motor performance of primary-grade school children. Research Quarterly, 22, 244260.

Sherar, L. B., Cumming, S. P., Eisenmann, J. C., Baxter-Jones, A. D. G., \&

Malina, R.

M. (2010). Adolescent biological maturity and physical activity: Biology meets behav- ior. Pediatric Exercise Science, 22, 332-349.

Sherar, L. B., Esliger, D. W., Baxter-Jones, A. D. G., \& Tremblay, M. S. (2007). Age and gender differences in youth physical activity: Does physical maturity matter? Medicine and Science in Sports and Exercise, 39, 830-835.

Skinner, R. A., \& Piek, J. P. (2001). Psychosocial implications of poor motor coordin- ation in children and adolescents. Human Movement Science, 20, 73-94.

Slaughter, M. H., Lohman, T. G., Boileau, R. A., Horswill, C. A., Stillman, R. J., Van Loan, M. D., ... Bemben, D. A. (1988). Skinfold equations for estimation of body fatness in children and youth. Human Biology; an International Record of Research, 60, 709-723.

Smart, J. E., Cumming, S. P., Sherar, L. B., Standage, M., Neville, H., \& Malina, R.

M. (2012). Maturity associated variance in physical activity and health-related quality of life in adolescent females: A mediated effects model. Journal of Physical Activity \& Health, 9(1), 86-95.

Stodden, D. F., Goodway, J. D., Langendorfer, S. J., Roberton, M. A., Rudisill, M. E., Garcia, C., ... Garcia, L. E. (2008). A developmental perspective on the role of motor skill competence in physical activity: An emergent relationship. Quest, 60, 290-306.

Strong, W. B., Malina, R. M., Blimkie, C. J. R., Daniels, S. R., Dishman, R. K., Gutin, B., ... Trudeau, F. (2005). Evidence based physical activity for school-age youth. The Journal of Pediatrics, 146, 732-737.

Thompson, A. M., Baxter-Jones, A. D. G., Mirwald, R. L., \& Bailey, D. A. (2003). Comparison of physical activity in male and female children: Does maturation matter?. Medicine and Science in Sports and Exercise, 35, 1684-1690.

Vandendriessche, J. B., Vandorpe, B., Coelho-e-Silva, M. J., Vaeyens, R., Lenoir, M., Lefevre, J., .. . Philippaerts, R. M. (2011). Multivariate association among morph- ology, fitness, and motor coordination characteristics in boys age 7 to 11. Pediatric Exercise Science, 23(4), 504-520.

Vandendriessche, J. B., Vandorpe, B. F. R., Vaeyens, R., Malina, R. M., Lefevre, J., Lenoir, M., .. . Philippaerts, R. M. (2012). Variation in sport participation, fitness and motor coordination with socioeconomic status among Flemish children. Pediatric Exercise Science, 24, 113-128. 
Vandorpe, B., Vandendriessche, J., Lefevre, J., Pion, J., Vaeyens, R., Matthys, S., .. . Lenoir, M. (2011). The korperkoordinationstest fur kinder: Reference values and suitability for 6-12-year-old children in Flanders. Scandinavian Journal of Medicine \& Science in Sports, 21(3), 378-388.

\section{Author Biographies}

Leonardo G. O. Luz is an Assistant Professor at Federal University of Alagoas, Brazil. Leonardo is currently a Phd-candidate at the University of Coimbra under the coordination of Manuel J Coelho-e-Silva and is being granted by the Brazilian Government (CAPES: BEX 1617/13-3)

Sean P. Cumming, after studying in the University of Edinburgh (Bsc, 1995), moved to the University of Exeter (Msc under the supervision of Stuart Biddle) and completed the $\mathrm{PhD}$ in Michigan State University (thesis: "Perceptions of adult autonomy support and self-determined motivation in recreational youth soccer: a bio-cultural perspective"). Additionally, he performed a post-doctoral program at the University of Washington under the supervision of Frank Smoll and Ronal Smith. He is now acting as Associate Professor in the School for Health, University of Bath, UK.

Joao P. Duarte completed a 3-year Bsc diplom in Sports Sciences by the University of Coimbra where he also obtained a 2-year master specialization in Sport Training. He is now PhD-candidate funded by the Portuguese Foundation for Science and Tecnology (SFRH/BD/101083/2014) and is cur- rently obtaining an extra 4-year diplom as Athletic Training. Joao Duarte was distinguished by Han Kemper with the Hans Stoboy award (the 2013 edition of Pediatric Work Physiology Meeting).

Joao Valente-dos-Santos, after obtaining a 5-year diplom in Sports Sciences, experienced a in-service training at the roller hockey department of Barcelona Football Club using Leonardo da Vinci program. Acted as Physical Educator while attending the master in Youth Sports by the University of Coimbra and was granted by the Portuguese Foundation for Science and Tecnhology: doc- toral studies (PhD: SFRH/BD/64648/2009, 2001-2014; Post-PhD: SFRH/BPD/

100470/2014, 2016-2017). Joao Valente-dos-Santos received the best poster award in the XXVIII edition of PWP, Children and Exercise).

Maria J. Almeida acted as Assistant Professor at the University of Madeira and is now experiencing a 5-year contract as Assistant Professor at the University of Coimbra.

Aristides Machado-Rodrigues completed his 5-year Bsc, 2-yr Msc and 4-year $\mathrm{PhD}$ in the University of Coimbra. He was granted by the Portuguese Foundation for Science and Technology (SRFH/BD/38988/2007). MachadoRodrigues received several awards by the Portuguese Society of Pediatric Obesity.

Cristina Padez is Assistant Professor in the Department of Anthropology, University of Coimbra and is acting as Head of the Research Unit in Anthropology and Health. 
Bruno Cleiton M. Carmo, LACAPS, Federal University of Alagoas, Brazil, is Assistant Professor at Federal University of Alagoas, Brazil.

Rute Santos, Early Start Research Institute, Faculty of Social Sciences, University of Wollongong, Australia; CIAFEL, University of Porto, Portugal.

Andre Seabra, $\mathrm{PhD}$ and Assistant Professor in the University of Porto and research member of CIAFEL. Seabra was granted by UEFA to implement a soccerbased intervention for obese children and youth that is receiving an impressive impact.

Manuel J. Coelho-E-Silva obtained his PhD under the supervision of Robert Malina supported by the Portuguese Foundation for Science and Technology and after studying in Michigan State University is now professor at the University of Coimbra (Portugal). He is teaching and conducting on going research in topics such as growth, maturation and talent; modelling perfor- mance; testing; pediatric ergometry; concurrent protocols including physical activity, biological maturation, body composition (field vs. laboratory; invasive vs. non-invasive). Chaired the 2013 edition of Pediatric Work Physiology Group. 